\title{
Letter \\ Cardiology, respiratory failure, and tolerance of hypoxia in the context of COVID-19: a multidisciplinary perspective
}

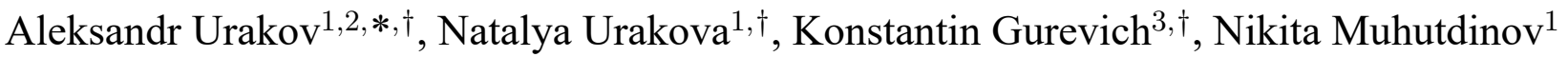 \\ ${ }^{1}$ Department of General and Clinical Pharmacology, Izhevsk State Medical Academy, 426034 Izhevsk, Udmurt Republic, Russia \\ ${ }^{2}$ Department of Modeling and Synthesis of Technological Structures, Institute of Mechanics, Udmurt Federal Research Center, 426067 Izhevsk, Udmurt \\ Republic, Russia \\ ${ }^{3}$ UNESCO Chair "Healthy lifestyle is the key to successful development", Moscow State Medical and Dental University, 127473 Moscow, Russia \\ *Correspondence: urakoval@live.ru (Aleksandr Urakov) \\ $\dagger$ These authors contributed equally. \\ Academic Editor: Peter A. McCullough \\ Submitted: 21 December 2021 Revised: 23 December 2021 Accepted: 23 December 2021 Published: 17 January 2022
}

\begin{abstract}
After reading with great interest the article entitled: "Exploring the impact of the COVID-19 pandemic on provision of cardiology services: a scoping review" redacted by Farah Yasmin et al., published by Reviews in Cardiovascular Medicine, we would like to add the following thoughts. Acute respiratory distress syndrome (ARDS) in Coronavirus disease 2019 (COVID-19) and pulmonary insufficiency reduces blood oxygen saturation and results in hypoxia. Therefore, the determining factor in the survival of patients with COVID-19 is their resistance to hypoxia. At the same time, it is the cardiovascular system that is an important and very sensitive link in the human adaptation to hypoxia. That is why it is necessary to carefully study the relationship between diseases of the heart, blood vessels, the reactivity of the cardiovascular system to hypoxia, and mortality in patients who develop ARDS with COVID-19.
\end{abstract}

Keywords: Hypoxia; Resistance; Adaptation; Acrocyanosis; Fingertips; Local hypothermia; Infrared imaging

After reading with great interest the article entitled: "Exploring the impact of the COVID-19 pandemic on provision of cardiology services: a scoping review" redacted by Farah Yasmin et al. [1], published by Reviews in Cardiovascular Medicine, we would like to add the following thoughts. Acute respiratory distress syndrome (ARDS) in COVID-19 and pulmonary insufficiency reduce blood oxygenation and result in hypoxia. Under these conditions, a patient's resistance to hypoxia is a determining factor in their survival during respiratory insufficiency in the final stage of atypical pneumonia. At the same time, it is the cardiovascular system that is an important and very sensitive link in the human adaptation to hypoxia. That is why it is necessary to carefully study not only the relationship between heart disease and mortality in ARDS [2,3], but also between the resistance of the cardiovascular system to hypoxia and mortality in COVID-19.

Firstly, COVID-19, due to the development of hypoxia, can provoke or worsen the severity of coronary heart disease. Secondly, hypoxia in COVID-19 can increase heart rate as an adaptation to hypoxia in order to increase blood supply to the brain. In this case, tachycardia can become a provoking and/or aggravating factor in heart failure. Thirdly, hypoxia during COVID-19 causes an adaptive change in the systemic circulation, known as redistribution of blood flow, in favor of vital organs and systems resulting in centralization of blood circulation. This change is accompanied by an increase in resistance to blood flow in peripheral blood vessels and is manifested by such phenom- ena as acrocyanosis and/or local hypothermia in the fingertips $[4,5]$.

It is quite possible that increased ischemic and hypoxic effects on the heart, which result in tachycardia and increased muscle activity of the heart, provoke and exacerbate underlying heart diseases during a coronavirus infection. Therefore, expanding the scope of research can improve the diagnosis and treatment of diseases of the cardiovascular system in SARS-CoV-2 [2,6].

There are reports that diseases of the cardiovascular system worsen in adults with hypoxia which develops when climbing high into the mountains, in patients with severe bronchial asthma and night apnea, as well as in fetuses who develop hypoxia during birth [7-11]. Moreover, in all these cases, the outcome is determined by the amount of human adaptation to hypoxia, and the ability to provide other sources of oxygen to the hypoxic patient. Assisted lung ventilation with supplemental oxygen and extracorporeal membrane oxygenation (ECMO) are the only reliable ways to save the lives of patients in the final stage of respiratory failure with COVID-19. Therefore, a multidisciplinary approach involving early application of an appropriate test (for example, the Stange test) to assess the patient's resistance to hypoxia, timely assessment of the patient's adaptation reserves to hypoxia, and analysis of individual reactivity of the cardiovascular system during voluntary apnea, can reduce the likelihood of cardiovascular complications and death in patients with COVID-19. 
The reaction of the cardiovascular system in patients with hypoxia varies as to whether there are underlying pathologies that are already present and varies from patient to patient. The prognosis worsens when heart pathology is already present. Infrared thermography and monitoring of the dynamics of the local temperature of the fingertips during voluntary apnea is the preferred method to assess the adaptation of the cardiovascular system and the entire patient's body to hypoxia. However, this method has only recently been instituted to assess hypoxia, and therefore; standard parameters for the diagnosis of hypoxia have not yet been developed. Nevertheless, infrared examination is possible for diagnostic purposes in hypoxic patients using such traditional tests as the Stange test and the cuff occlusion test. However, diagnostic criteria for the dynamics of local temperature changes of the fingertips in the conditions of standard ischemia and hypoxia have not yet been determined. Therefore, further studies are needed to determine the relationship between the ability of patients with diseases of the cardiovascular system to adapt to periods of hypoxia, and its relationship to mortality in patients who develop ARDS with COVID-19.

\section{Author contributions}

$\mathrm{AU}, \mathrm{NU}$ and $\mathrm{KG}$ designed and performed the research study, NM were in charge of the correction of the language and writing. All authors contributed to editorial changes in the manuscript. All authors read and approved the final manuscript.

\section{Ethics approval and consent to participate}

Not applicable.

\section{Acknowledgment}

We thank Antoniy Nowakowsky, Kurt Ammer and Yin Kwee Ng for their spiritual support and timely valuable advice.

\section{Funding}

This research received no external funding.

\section{Conflict of interest}

The authors declare no conflict of interest.

\section{References}

[1] Yasmin F, Shujauddin SM, Naeem A, Jabeen A, Shah SMI, Ochani RK, et al. Exploring the impact of the COVID-19 pandemic on provision of cardiology services: a scoping review. Reviews in Cardiovascular Medicine. 2021; 22: 83-95.

[2] Corvera CZ, Muente-Alva LS. Cardiology and respiratory failure in the context of COVID-19: a multidisciplinary perspective. Reviews in Cardiovascular Medicine. 2021; 22: 547-548.

[3] Jayasimhan D, Foster S, Chang CL, Hancox RJ. Cardiac biomarkers in acute respiratory distress syndrome: a systematic review and meta-analysis. Journal of Intensive Care. 2021; 9: 36.

[4] Copeman PW. Acrocyanosis: a blood disease? Proceedings of the Royal Society of Medicine. 1973; 66: 741-742.

[5] Urakov A, Kasatkin A, Ammer K, Gurevich K. The dynamics of fingertip temperature during voluntary breath holding and its relationship to transcutaneous oximetry. Thermology International. 2019; 29: 65-66.

[6] Nishiga M, Wang DW, Han Y, Lewis DB, Wu JC. COVID-19 and cardiovascular disease: from basic mechanisms to clinical perspectives. Nature Reviews Cardiology. 2020; 17: 543-558.

[7] Penaloza D, Arias-Stella J. The heart and pulmonary circulation at high altitudes: healthy highlanders and chronic mountain sickness. Circulation. 2007; 115: 1132-1146.

[8] Pratali L, Allemann Y, Rimoldi SF, Faita F, Hutter D, Rexhaj E, et al. RV Contractility and Exercise-Induced Pulmonary Hypertension in Chronic Mountain Sickness. JACC: Cardiovascular Imaging. 2013; 6: 1287-1297.

[9] Xu M, Xu J, Yang X. Asthma and risk of cardiovascular disease or all-cause mortality: a meta-analysis. Annals of Saudi Medicine. 2017; 37: 99-105.

[10] Drager LF, McEvoy RD, Barbe F, Lorenzi-Filho G, Redline S. Sleep Apnea and Cardiovascular Disease: Lessons from Recent Trials and need for Team Science. Circulation. 2017; 136: 1840 1850.

[11] Giussani DA, Niu Y, Herrera EA, Richter HG, Camm EJ, Thakor AS, et al. Heart disease link to fetal hypoxia and oxidative stress. Advances in Experimental Medicine and Biology. 2014; 814: 77-87. 